\title{
Uso da ultrassonografia no manejo da via aérea difícil
}

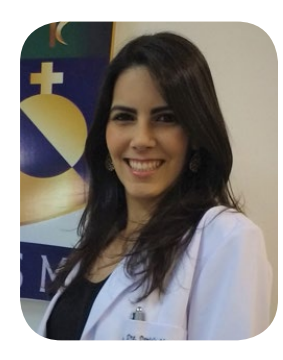

\author{
Daniela Nunes Oliveira', Catharina Borges de Oliveira', Murilo
}

Pereira Flores ${ }^{1}$

\section{RESUMO}

O manejo adequado da via aérea é fundamental para a segurança do paciente e prevenção de complicações graves durante 0 ato anestésico. A avaliação pré-anestésica ajuda a identificar preditores de risco para via aérea difícil e, desta maneira, contribui na escolha da técnica anestésica mais adequada ao paciente. Neste contexto, o uso da ultrassonografia, como um método acessível, não invasivo e prático, vem como ferramenta adicional à avaliação da via aérea. Este artigo trata-se de uma revisão da literatura que tem como objetivo apresentar os fundamentos teóricos e aspectos práticos do uso da ultrassonografia como ferramenta auxiliar no manejo da via aérea potencialmente difícil. Foram descritas as técnicas para identificação das diferentes estruturas anatômicas envolvidas na abordagem das vias aéreas, suas aplicações práticas, bem como as evidências científicas que as fundamentam. Além disso, a ultrassonografia também tem aplicabilidade na confirmação do adequado posicionamento do tubo endotraqueal, localização de nervos periféricos para guiar bloqueios anestésicos úteis para intubação acordado e na avaliação de resíduo gástrico para prever o risco de broncoaspiração. Assim sendo, a ultrassonografia, apesar de ser um instrumento relativamente recente na avaliação da via aérea, tem se mostrado um método útil e rápido para auxiliar na predição de via aérea difícil e, desta maneira, prevenir algumas complicações durante o ato anestésico. Apesar da necessidade de estudos maiores para reforçar as medidas ultrassonográficas consideradas de risco, a associação de parâmetros clínicos aumenta a acurácia do método e resulta em maior segurança para o paciente.

KEY-WORDS: airway management; ultrasonography; difficult airway

\section{INTRODUÇÃO}

A abordagem à via aérea faz parte da rotina dos anestesiologistas e, apesar disso, incidentes críticos durante a anestesia são geralmente relacionados ao manejo da via aérea. De acordo com o $4^{\circ}$ Relatório do National Audit Project of the Royal College of Anaesthetists and Difficult Airway Society (NAP4), a incidência de complicações graves relacionadas à via aérea foi de 1 a cada 22.000 anestesias gerais. Admissão não programada em unidade de tratamento intensivo aconteceu em 1 a cada 29.000 e necessidade de via aérea cirúrgica, em 1 a cada 50.000 anestesias gerais. Morte ou lesão cerebral ocorreram em 1 a cada 180.000 anestesias. $^{1}$

A avaliação acurada da via aérea deveria sempre ser realizada no preparo pré-anestésico, de forma a identificar pacientes com potencial via aérea difícil e planejar a forma mais segura e adequada de abordagem da mesma. Via aérea difícil não prevista permanece um desafio para o anestesiologista, especialmente quando associada à ventilação difícil. Apesar da existência de vários parâmetros clínicos (Classificação de Mallampati, distância interincisivos, distância tireomentoniana, índice de massa corpórea), isoladamente, os mesmos têm apresentado baixa sensibilidade e especificidade, com limitado valor preditivo. Melhoria na capacidade de predição ocorre com a associação desses múltiplos parâmetros. ${ }^{2}$

O uso da ultrassonografia para a avaliação da via aérea tem sido proposto, recentemente, como uma ferramenta adicional para melhorar a identificação e abordagem à via aérea difícil. Os principais pontos positivos dessa ferramenta são a relativa simplicidade, disponibilidade à beira do leito e por tratar-se de método não-invasivo. ${ }^{2}$ Vários estudos têm sido realizados para identificar medidas ultrassonográficas específicas associadas à ocorrência de via aérea difícil. 
Este artigo trata-se de uma revisão da literatura com o objetivo de apresentar os fundamentos teóricos e aspectos práticos do uso da ultrassonografia como uma ferramenta auxiliar no manejo da via aérea potencialmente difícil.

\section{IDENTIFICAÇÃO DAS ESTRUTURAS ANATÔMICAS Língua}

Com o paciente em decúbito dorsal e o pescoço estendido, mantendo a boca fechada e a língua tocando os dentes incisivos, posiciona-se o transdutor sob o queixo no plano sagital mediano. ${ }^{3}$ (Imagem 1 )

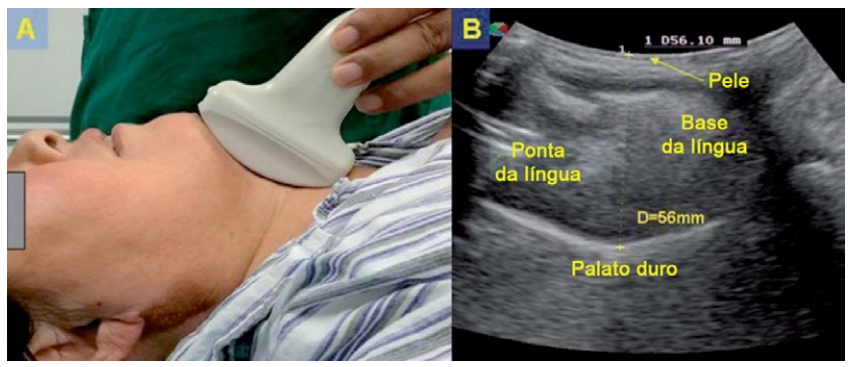

Figura 1 - Identificação da língua: A) Posição do transdutor; B) Posição da língua.

\section{Epiglote e Cordas Vocais}

Com o transdutor posicionado transversalmente na região da membrana tireoidea e deslocando-se caudalmente sobre a cartilagem tireoide, visualiza-se a epiglote (estrutura linear hipoecoica) e as cordas vocais (estruturas hiperecoicas em forma de V). Manobras como a protrusão da língua e deglutição ajudam na identificação da epiglote, assim como movimentos respiratórios e fonação facilitam na identificação das cordas vocais. ${ }^{2}$ (Imagem 2 )

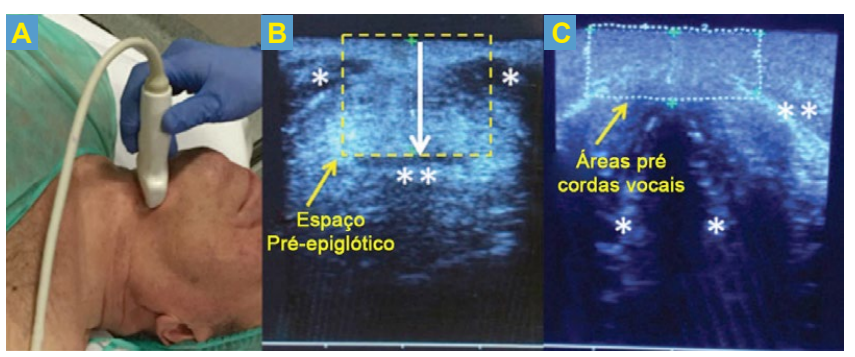

Figura 2 - Identificação da epiglote e cordas vocais: A) Posição do transdutor; B) Epiglote; C) Cordas vocais.

\section{Membrana Cricotireoidea}

1- Abordagem Transversa: técnica TACA ("cartilagem tireoide - ar - cartilagem cricoide - $\left.a r^{\prime \prime}\right)^{4}$
Posiciona-se transversalmente o transdutor na região anterior do pescoço, ao nível da cartilagem tireoide, movendo-o até que a mesma seja visualizada como uma estrutura triangular hiperecoica ("branca"). Direciona-se caudalmente o transdutor até a identificação da membrana cricotireoidea (estrutura linear hiperecoica, resultante da interface ar-tecido). $O$ transdutor é direcionado mais caudalmente até a identificação da cartilagem cricoide ("faixa preta em formato de C" com uma linha branca em sua curvatura). Por fim, desloca-se novamente o transdutor em direção cefálica até a identificação da membrana cricotireoidea. (Imagem 3)

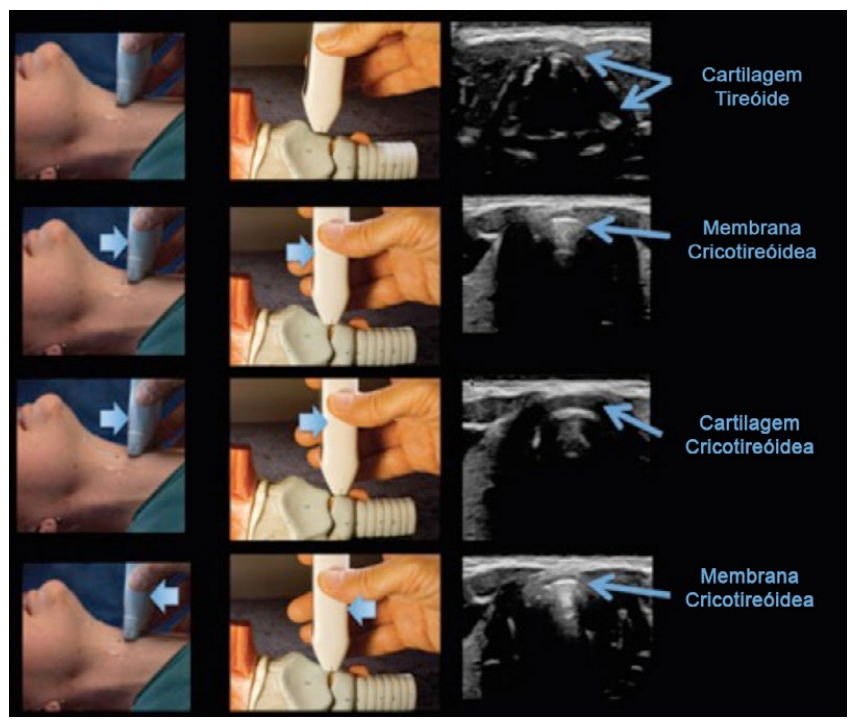

Figura 3 - Identificação da Membrana Cricotireoidea Abordagem Transversa (Técnica TACA).

\section{2- Abordagem Longitudinal - Técnica do "Cordão de} Pérolas"4

a) Palpa-se o nó supraesternal e, no pescoço, acima do mesmo, posiciona-se o transdutor transversalmente para visualização da traqueia (estrutura hipoecoica em formato de ferradura com uma linha hiperecoica posteriormente).

b) Desloca-se o transdutor à direita da traqueia, de forma que a borda direita do transdutor esteja posicionada sobre a linha média da mesma.

c) Mantendo a borda direita do transdutor sobre a linha média, faz-se uma rotação de $90^{\circ}$ na borda esquerda, com consequente posicionamento longitudinal do transdutor sobre a linha média da traqueia. Os anéis traqueais serão vistos como estruturas pretas (hipoecoicas), localizadas à frente de uma linha branca (hiperecoica), formando uma estrutura semelhante a um cordão de pérolas. 
d) Desloca-se cefalicamente o transdutor, mantendo-o na posição longitudinal, até a visualização da cartilagem cricoide e a porção distal da cartilagem tireoide. A membrana cricotiroidea localiza-se entre essas duas estruturas. (Imagem 4)

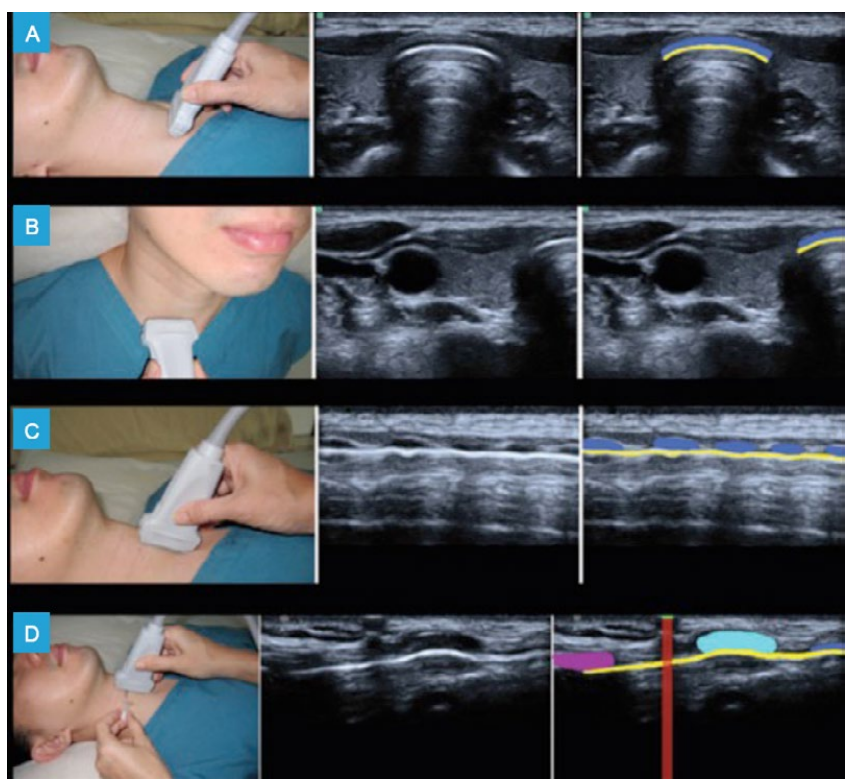

Figura 4 - Identificação da Membrana Cricotireoidea - Técnica do "Cordão de Pérolas": A) Identificação da traqueia; B) Lateralização do transdutor; C) Visualização longitudinal da traqueia ("Cordão de Pérolas"); D) Membrana cricotireoidea (vermelho) entre a cartilagem tireoidea (roxo) e a cartilagem cricoide (azul).

\section{AVALIAÇÃO ULTRASSONOGRÁFICA DA VIA AÉ- REA DIFÍCIL: QUAIS AS EVIDÊNCIAS CIENTÍFICAS?}

Baseados no pressuposto de que o aumento da espessura da língua dificulta a laringoscopia e a intubação traqueal, com consequente aumento do risco de via aérea difícil, Yao et. al. ${ }^{3}$ realizaram medidas ultrassonográficas da espessura lingual em 2.480 pacientes e as correlacionaram com dificuldade de intubação e laringoscopia. Eles concluíram que a espessura da língua avaliada por ultrassonografia foi um preditor independente para intubação traqueal e laringoscopia difíceis, assim como o aumento da razão entre espessura da língua e distância tireomentoniana. Espessura da língua maior que $6,1 \mathrm{~cm}$ foi associada à intubação difícil com sensibilidade de $75 \%$ e especificidade de $72 \%$ (Oddsratio igual a 7,7), enquanto que a razão entre espessura da língua e distância tireomentoniana superior a 0,87 apresentou sensibilidade de $84 \%$ e especificidade de $79 \%$ (Oddsratio de 20).
Gupta et. al. ${ }^{5}$ demonstraram correlação entre a razão das distâncias do espaço pré-epiglótico e epiglote-cordas vocais [Pre-E/E-VC] e a classificação de Cormack-Lehane $(\mathrm{CL})$. Valores [Pre-E/E-VC] maiores que 0 e menores que 1 correlacionaram-se com CL grau 1; [Pre-E/E-VC] entre 1 e 2, correlacionaram-se com CL grau 2; e valores [Pre-E/E-VC] entre 2 e 3, com CL grau 3. A sensibilidade desse método foi de $67 \%-68 \%$, com rápida aquisição ultrossonográfica das imagens e medidas (aproximadamente 30 segundos).

Rana et. al. ${ }^{6}$ reforçaram esse achado ao concluir que a medida ultrasonográfica [Pre-E/E-VC] é um bom preditor para avaliar laringoscopia fácil ou difícil no pré-operatório, com boa correlação com a Classificação de Cormack-Lehane. A [Pre-E/E-VC] tem alta previsibilidade com um ponto de corte $>1,77$ para laringoscopia difícil.

Estudo conduzido por Falcetta et. al. ${ }^{2}$ evidenciou que medidas ultrassonográficas da espessura dos tecidos moles, na face anterior do pescoço, em dois níveis - membrana tireoidea (espaço pré-epiglótico) e cordas vocais - correlacionam-se fidedignamente com a Classificação de Cormack-Lehane, com consequente predição de via aérea difícil. Medidas do espaço pré-epiglótico superiores a $2,54 \mathrm{~cm}$ tiveram sensibilidade de $82 \%$ e especificidade de $91 \%$ para predizer laringoscopia difícil (CL>2b).

Acricotireoidotomia é considerada o último recurso para o manejo da via aérea difícil. Apesar disso, as taxas de sucesso para identificação dessa estrutura através de inspeção e palpação, por parte dos anestesiologistas, são extremamente baixas, principalmente em indivíduos obesos. Kristensen et. al. ${ }^{7}$ compararam o método de palpação digital e o uso de ultrassonografia para identificação da membrana cricotireoidea em paciente obesa mórbida. Quando o método convencional de palpação digital foi utilizado, 13 dos 35 anestesiologistas participantes (37\%) foram capazes de localizar a membrana cricotireoidea com sucesso, em 2 minutos, enquanto 29 dos 35 participantes (83\%) identificaram corretamente a estrutura com auxílio do ultrassom. Esses mesmos autores ${ }^{8}$ compararam duas técnicas ultrassonográficas para a localização da membrana cricotireoidea: a técnica transversal (TACA) e a técnica longitudinal ("Cordão de Pérolas"). E concluiu que a técnica transversal foi mais rápida (média= $24 \mathrm{~s}$ ) do que a longitudinal (média $=37,6 \mathrm{~s}$ ). Trinta e oito $(90 \%)$ dos 42 anestesiologistas que participaram desse estudo identificaram corretamente a membrana cricotireoidea usando cada uma das técnicas. Todos os participantes acertaram a localização dessa estrutura 
com, ao menos, uma das técnicas. Devido à velocidade de identificação, a técnica transversal parece ser útil também em situações emergenciais, assim como em casos onde não há espaço suficiente para o adequado posicionamento longitudinal do transdutor (ex.: pacientes com pescoço curto ou deformidade que impossibilite a extensão do pescoço).

Corroborando essa aplicabilidade, Okano e colaboradores ${ }^{9}$ relataram o caso de uma paciente com grande abscesso cervical desviando lateralmente a traqueia. A localização da membrana cricotireoidea, para realização de cricotireoidotomia, foi possível apenas com o uso da técnica ultrassonográfica transversal (TACA). Devido a situações como essa, Kristensen sugere, em revisão, que a membrana cricotireoidea seja identificada em todos os pacientes antes da indução anestésica e em todos os pacientes com comprometimento da via aérea se o tempo permitir, inicialmente através da inspeção seguida pela palpação e, em caso de falha ou dúvida, através da ultrassonografia.

\section{OUTRAS APLICAÇÕES \\ Confirmação do adequado posicionamento do tubo endotraqueal}

A confirmação da intubação traqueal pode ser feita diretamente, através de escaneamento em tempo real, durante a intubação, ou indiretamente, através da observação da expansão pulmonar e diafragmática. Visualização direta da passagem da cânula traqueal tem a vantagem de reconhecimento imediato de intubação esofágica, antes que a ventilação seja iniciada com consequente insuflação de ar para o estômago, reduzindo o risco de êmese e broncoaspiração. Ambas as técnicas são superiores à capnografia em situações de muito baixo fluxo sanguíneo. ${ }^{10}$

A técnica direta pode ser realizada longitudinalmente ao nível da membrana cricotireoidea ou, através da visualização da traqueia, com o transdutor posicionado transversalmente acima do nó supraesternal. Essa técnica apresentou uma sensibilidade de $97 \%$ para o correto diagnóstico de intubação esofágica quando realizada durante a intubação, dinamicamente. Quando realizada após a intubação, a sensibilidade foi consideravelmente menor. ${ }^{10}$

A técnica indireta é realizada por meio de ultrassonografia pulmonar, através da visualização da linha pleural. Sua principal vantagem é a identificação de intubação seletiva. O movimento sincrônico de "deslizamento pleural" é visualizado durante a ventilação espontânea ou controlada. Em contrapartida, durante períodos de apneia, nenhum deslizamento pleural é ob- servado, mas, sim, pequenos movimentos sincrônicos ao batimento cardíaco, chamado de "pulso pulmonar". A distinção entre intubação traqueal ou endo-brônquica pode ser feita através de ultrassonografia em ambos os pulmões. Se há deslizamento pleural de um lado e pulso pulmonar do outro, isso indica que o tubo está posicionado no brônquio fonte do lado que é observado o deslizamento pulmonar. ${ }^{10}$

\section{Bloqueios anestésicos úteis para intubação acordada}

A ultrassonografia tem sido largamente usada para localização de vários nervos e realização de bloqueios anestésicos. Redução da quantidade de anestésicos, aumento das taxas de sucesso e redução do número de complicações são os principais benefícios do uso da ultrassonografia nesse contexto. $\mathrm{Na}$ abordagem à via aérea, a realização de bloqueios anestésicos é importante no preparo para intubação acordada, técnica padrão ouro para intubação de pacientes com via aérea difícil e jejum incompleto.

Manikandan et. al. ${ }^{11}$ descreveram o uso da ultrassonografia para guiar o bloqueio do nervo laríngeo superior bilateralmente no preparo para intubação acordada em paciente que se submeteria à artrodese cervical de urgência. O corno maior do osso hioide e a artéria laríngea superior foram localizados e anestésico local foi injetado entre essas duas estruturas.

A identificação da membrana cricotireoidea descrita previamente torna-se útil ainda para a administração transtraqueal de anestésicos para anestesia da traqueia e laringe abaixo das cordas vocais.

\section{Avaliação de resíduo gástrico}

A ultrassonografia também tem validação e aplicabilidade na avaliação do resíduo gástrico. Sabe-se que a presença de conteúdo gástrico durante a indução anestésica (pela perda do reflexo de proteção de via aérea e relaxamento do esfíncter esofagiano inferior) aumenta o risco de broncoaspiração e, consequentemente, a morbimortalidade associada ao ato anestésico.

Neste contexto, o uso da ultrassonografia, por se tratar de um método de baixo custo, portátil e prático, permite o exame do conteúdo gástrico à beira do leito e, desta maneira, ajuda na escolha da melhor técnica anestésica para o manejo das vias aéreas, reduzindo o risco de broncoaspiração. ${ }^{12}$

Apesar das limitações do método, como o fato de ser operador e aparelho dependentes e não avaliar o pH do conteúdo gástrico, a ultrassonografia permite a avaliação do volume e natureza do material contido no estômago. 
A técnica utilizada compreende uma análise quantitativa (área da secção transversa do antro gástrico e volume total do estômago) e qualitativa. Nesta última é feita uma avaliação do antro gástrico e o estômago é classificado como: a) vazio (se paredes anterior e posterior justapostas); b) endocavidade com conteúdo líquido (paredes distendidas e conteúdo hipoecoico); c) endocavidade com conteúdo sólido (paredes distendidas com imagem em "vidro fosco" ou semelhante ao parênquima hepático). ${ }^{12,13}$

\section{CONCLUSÃO}

A aplicabilidade da ultrassonografia em anestesiologia tem sido expandida, tornando-se um instrumento extremamente importante no cotidiano do anestesiologista. Seu uso na abordagem da via aérea, apesar de relativamente recente, tem se mostrado seguro, rápido e reproduzível. Está bem estabelecida a adequada identificação da membrana cricotireoidea ${ }^{7,8,9}$, importante referência para abordagem cirúrgica emergencial da via aérea, assim como a confirmação do adequado posicionamento da cânula traqueal, tanto de forma direta como indireta. ${ }^{10}$ Além disso, a ultrassonografia também é validada no contexto da avaliação de resíduo gástrico, com o objetivo de prevenir a broncoaspiração através da escolha da técnica anestésica e manejo de via aérea adequados ao paciente..$^{12,13}$

Em relação à capacidade de predição de via aérea difícil pelas medidas ultrassonográficas apresentadas acima, faz-se necessária a realização de estudos maiores para reforçarem esses achados. A associação dessas medidas diagnósticas com os parâmetros clínicos já utilizados, para avaliação da via aérea, pode melhorar a acurácia da avaliação perioperatória para a identificação de via aérea difícil. Isso resultará em maior segurança, ao evitar surpresas desagradáveis durante a abordagem da via aérea na sala operatória.

\section{REFERÊNCIAS}

1. Cook TM, Woodall N, Frerk C. Major complications of airway management in the UK: results of the Fourth $\mathrm{Na}$ tional Audit Project of the Royal College of Anaesthetists and the Difficult Airway Society. Part 1: Anaesthesia. Br J Anaesth 2011; 106:617-31.

2. Falcetta S, Cavallo S, Gabbanelli V, Pelaia P, Sorbello $\mathrm{M}$, Zdravkovic I, Donati A. Evaluation of two neck ultrasound measurements as predictors of difficult direct laryngoscopy. A prospective observational study. Eur J Anaesthesiol 2018; 35:605-612.

3. Yao W, Wang B. Can tongue thickness measured by ultrasonography predict difficult tracheal intubation? $\mathrm{Br}$
J Anaesth 2017; 118:601-9.

4. Kristensen MS, Teoh WH, Rudolph SS. Ultrasonographic identification of the cricothyroid membrane: best evidence, techniques, and clinical impact. $\mathrm{Br} \mathrm{J}$ Anaesth 2016;117:i39-i48.

5. Gupta D, Srirajakalidindi A, Ittiara B, Apple L, Toshniwal $\mathrm{G}$, Harber $\mathrm{H}$. Ultrasonographic modification of Cormack-Lehane Classification for pre-anesthetic airway assessment. Middle East J Anesth 2011; 21:835-42.

6. Rana S, Verma V, Bhandari S, Sharma S, Koundal V, Chaundhary SK. Point-of-care ultrasound in the airway assessment: A correlation of ultrasonography-guided parameters to the Cormack-Lehane Classification. Saudi J Anaesth 2018; 12:292-6.

7. Kristensen MS, Teoh WH, Rudolph SS, Tvede MF, Hesselfeldt R, Borglum J, Lohse T, Hansen LN. Structured approach to ultrasound-guided identification of the cricothyroid membrane: a randomized comparasion with the palpation method in the morbidly obese. $\mathrm{Br} J$ Anaesth 2015;114:1003-4.

8. Kristensen MS, Teoh WH, Rudolph SS, Hesselfeldt R, Borglum J, Tvede MF. A randomized cross-over comparison of the transverse and longitudinal techniques for ultrasound-guided identification of the cricothyroid membrane in morbidly obese subjects. Anaesthesia 2016;71:675-83.

9. Okano H, Uzawa K, Watanabe K, Motoyasu A, Tokumine J, Lefor AK, Yorozu T. Ultrasound-guided identification of the cricothyroid membrane in a patient with a difficult airway: a case report. BMC Emergency Medicine 2018;18:5.

10. Kristensen MS. Ultrasonography in the management of the airway. ActaAnaesthesiolScand 2011;55:1155-73.

11. Manikandan S, Neema PK, Rathod RC. Ultrasound-guided bilateral superior laryngeal nerve block to aid awake endotracheal intubation in a patient with cervical spine disease for emergency surgery. Anaesth Intensive Care 2010; 38:946-48.

12. Bisinotto FMB, Naves AA, Lima HM, Peixoto ACA, Maia GC, Junior PPR, Martins LB, Silveira LAM. Use ofultrasound for gastric volume evaluation after ingestion of different volumes of isotonic solution. Brazilian Journal of Anesthesiology (EnglishEdition) 2017;67(4):376-382.

13. Bisinotto FMB, Pansani PL, Silveira LAM, Naves AA, Peixoto ACA, Lima HM e et. al. Qualitative and quantitative ultrasound assessment of gastriccontent. Rev. Assoc. Med. Bras 2017;63(2):134-141.

1- Serviço de Anestesiologia do HSI. Endereço para correspondência: danih.oliveira@hotmail.com 